Women's hormone levels modulate the motivational salience of facial attractiveness and sexual dimorphism

Short title: Hormones, motivation, and faces

Hongyi Wang ${ }^{1^{*}}$, Amanda C. Hahn ${ }^{1^{*}}$, Claire I. Fisher ${ }^{1}$, Lisa M. DeBruine ${ }^{1}, \&$ Benedict C. Jones ${ }^{1}$

1. Institute of Neuroscience and Psychology, University of Glasgow, Glasgow, G12 8QB, UK.

* Joint first authors

Corresponding author

Hongyi Wang, Institute of Neuroscience and Psychology, University of Glasgow, Glasgow, G12 8QB, UK.

Tel: 011441413305089

Email: h.wang.4@research.gla.ac.uk 


\section{Women's hormone levels modulate the motivational salience of facial attractiveness and sexual dimorphism}

\section{Abstract}

The physical attractiveness of faces is positively correlated with both behavioral and neural measures of their motivational salience. Although previous work suggests that hormone levels modulate women's perceptions of others' facial attractiveness, studies have not yet investigated whether hormone levels also modulate the motivational salience of facial characteristics. To address this issue, we investigated the relationships between within-subject changes in women's salivary hormone levels (estradiol, progesterone, testosterone, and estradiol-to-progesterone ratio) and within-subject changes in the motivational salience of attractiveness and sexual dimorphism in male and female faces. The motivational salience of physically attractive faces in general and feminine female faces, but not masculine male faces, was greater in test sessions where women had high testosterone levels. Additionally, the reward value of sexually dimorphic faces in general and attractive female faces, but not attractive male faces, was greater in test sessions where women had high estradiol-to-progesterone ratios. These results provide the first evidence that the motivational salience of facial attractiveness and sexual dimorphism is modulated by within-woman changes in hormone levels.

Keywords: mate preference; incentive salience; reward; attractiveness; menstrual cycle; testosterone 


\section{Introduction}

Facial attractiveness is a particularly salient social cue that influences many important social outcomes. For example, people prefer to mate with, date, associate with, hire, and vote for attractive individuals (see Langlois et al., 2000 for a meta-analytic review). Several lines of evidence also demonstrate that physically attractive faces have motivational salience. For example, the extent to which people will key press to increase the length of time for which they can view faces is correlated with the physical attractiveness of the faces (Aharon et al., 2001; Levy et al., 2008; Hahn et al., 2013). Additionally, compared to viewing physically unattractive faces, viewing physically attractive faces elicits greater activation in brain regions implicated in motivation and the processing of rewards, such as the nucleus accumbens and medial orbitofrontal cortex (see Bzdok et al., 2011 and Mende-Siedlecki et al., 2013 for meta-analytic reviews). Moreover, behavioral measures of motivational salience predict neural measures of faces' reward value better than do perceptions of attractiveness measured by ratings (Aharon et al., 2001).

Several lines of evidence suggest that changes in women's hormone levels during the menstrual cycle may affect their perceptions of others' facial attractiveness (see Gildersleeve et al., 2014 for a meta-analytic review). For example, studies have reported that women's preferences for masculine men are positively correlated with their estradiol (e.g., Roney \& Simmons, 2008; Roney et al., 2011) or testosterone (e.g., Welling et al., 2007; Bobst et al., 2014) levels. By contrast with the relatively large number of studies 
investigating how women's perceptions of others' attractiveness covary with changes in women's hormone levels, no previous studies have tested for effects of women's hormone levels on the motivational salience of facial attractiveness. This is surprising, given the importance of attractiveness for social interaction (Langlois et al., 2000) and research suggesting that women's testosterone (Hermans et al., 2010) or estradiol (Dreher et al., 2007) modulates the extent to which financial incentives activate brain regions involved in motivation and the processing of reward.

In light of the above, we investigated the hormonal correlates of within-woman changes in the motivational salience of male and female facial attractiveness. Women (none of whom were using any form of hormonal supplement, such as hormonal contraceptives) were each tested once a week for five weeks (i.e., each woman completed five weekly test sessions). In each of these test sessions, the motivational salience of male and female facial attractiveness was assessed and a saliva sample was collected. The motivational salience of faces was measured using a standard key-press task that has previously been shown to be a particularly good predictor of neural measures of the reward value of faces (Aharon et al., 2001). Saliva samples were analyzed for estradiol, progesterone, and testosterone levels.

Many previous studies of hormone-mediated responses to faces have emphasized the potential importance of sexually dimorphic facial characteristics, particularly in men's faces (reviewed in Gildersleeve et al., 2014). Since the relationship between men's facial attractiveness and sexual 
dimorphism is complex (reviewed in Roney et al., 2011), with many studies finding no correlation between sexual dimorphism and attractiveness, we also tested for possible effects of hormone levels on the motivational salience of sexual dimorphism in faces. Given that sexual dimorphism and attractiveness are more reliably and highly correlated in female than male faces (see Rhodes, 2006 for a meta-analytic review), the effects of hormone levels on responses to attractiveness and sexual dimorphism in female faces may be more similar than the corresponding effects for male faces.

\section{Methods}

\subsection{Participants}

Fifty heterosexual women (mean age $=21.2$ years, $S D=2.89$ years) participated in the study. All participants were students at the University of Glasgow (Scotland, UK). None of these women were currently pregnant, breastfeeding, or taking any form of hormonal supplement, and all indicated that they had not taken any form of hormonal supplement in the 90 days prior to participation. All participants provided written, informed consent.

\subsection{Face stimuli}

Stimuli were full-color face images of 50 white adult men (mean age $=24.2$ years, $S D=3.99$ years) and 50 white adult women (mean age $=24.3$ years, $\mathrm{SD}=4.01$ years). Photographs were taken under standardized photographic conditions and depicted individuals who were posed front on to the camera with neutral emotional expressions and direct gaze. Images were aligned on pupil position and masked so that clothing was not visible. 
In order to establish the attractiveness of the faces for comparison with motivational salience, the 50 male faces were rated for attractiveness by 100 heterosexual women and 100 heterosexual men (mean age $=24.7$ years, $\mathrm{SD}=5.87$ years) using a 1 (much less attractive than average) to 7 (much more attractive than average) scale. A different set of 100 heterosexual women and 100 heterosexual men (mean age $=25.0$ years, $S D=5.56$ years) rated the 50 female faces using the same 7-point scale. Participants were randomly allocated to rate either male or female faces. Trial order within each block was fully randomized.

Inter-rater agreement was high for attractiveness ratings of both the male (Cronbach's $\alpha=.99$ ) and female (Cronbach's $\alpha=.99)$ faces. Because mean ratings derived from female and male raters' scores were highly correlated for both male $(r=.97, \mathrm{~N}=50, p<.001)$ and female $(r=.96, \mathrm{~N}=50, p<.001)$ faces, we combined ratings from female and male raters to produce a single attractiveness score for each face. These facial attractiveness scores were used in our main analyses (see Results).

In order to establish the sexual dimorphism of the faces for comparison with motivational salience, the 50 male faces were rated for masculinity by 100 heterosexual women and 100 heterosexual men (mean age=24.4 years, $\mathrm{SD}=5.32$ years) using a 1 (much less masculine than average) to 7 (much more masculine than average) scale. A different set of 100 heterosexual women and 100 heterosexual men (mean age $=24.3$ years, $S D=5.19$ years) 
rated the 50 female faces for femininity using a 1 (much less feminine than average) to 7 (much more feminine than average) scale. Other than the rating scale used, the procedure was identical to the procedure used to collect attractiveness ratings.

Inter-rater agreement was high for sexual dimorphism ratings of both the male (Cronbach's $\alpha=.99$ ) and female (Cronbach's $\alpha=.99$ ) faces. Because mean ratings derived from female and male raters' scores were highly correlated for both male $(r=.97, \mathrm{~N}=50, p<.001)$ and female $(r=.97, \mathrm{~N}=50, p<.001)$ faces, we combined ratings from female and male raters to produce a single sexual dimorphism score for each face. These sexual dimorphism scores were used in our main analyses (see section 3).

Attractiveness and sexual dimorphism ratings were highly correlated for female faces $(r=.86, \mathrm{~N}=50, p<.001)$. The correlation between attractiveness and sexual dimorphism ratings for male faces $(r=.31, \mathrm{~N}=50, p=.031)$ was significantly weaker (Fisher r-to-z transformation: $z=4.72, p<.001$ ).

\subsection{Procedure}

In order to investigate how hormone levels might modulate the motivational salience of faces, each of the 50 women in our main study completed five weekly test sessions. During each test session, participants provided a saliva sample via passive drool (Papacosta \& Nassis, 2011). Each woman's test sessions took place at the same time of day to control for possible effects of diurnal changes in hormone levels (Veldhuis et al., 1988; Bao et al., 2003). 
In each test session, participants completed two versions of a standard keypress task, similar to those used to assess the motivational salience of faces in previous studies (Aharon et al., 2001; Levy et al., 2008; Hahn et al., 2013). Following Aharon et al. (2001) and Levy et al. (2008), and because the faces had been rated in single-sex blocks (see section 2.2), male and female faces were presented in separate blocks of trials. In one version of the task (male face version), the 50 male faces described in section 2.2 were presented in a fully randomized order. In the other version of the task (female face version), the 50 female faces described in section 2.2 were presented, again in a fully randomized order. Within each test session, participants completed the male face version of the task and the female face version in a random order.

In each version of the key-press task, participants controlled the viewing duration of each face image by repeatedly pressing designated keys on their keyboard after initiating each trial by pressing the space bar. Participants could increase the length of time a given face was displayed by alternately pressing the 7 and 8 keys and/or decrease the length of time a given face was displayed by alternately pressing the 1 and 2 keys. Each key press increased or decreased the viewing duration by $100 \mathrm{~ms}$. The default viewing duration for each image (i.e., the length of time a face remained onscreen if no keys were pressed) was 4 seconds. Participants were told that the key-press task would last for a total of 3.5 minutes in order to discourage responses aimed at changing the length of engagement with the task. However, in reality, the total length of the key-press task was dependent on participants' responses. All 
participants key-pressed at least once in each version of the task in all test sessions. Participants completed a block of practice trials at the start of each test session to ensure they understood the task (face images were not shown in this block of practice trials).

Following previous studies of the motivational salience of faces (Aharon et al., 2001; Levy et al., 2008; Hahn et al., 2013), key-press scores for each face were calculated by subtracting the number of key presses made to decrease viewing time from those made to increase viewing time. These scores were calculated separately for each participant and for each test session and served as the dependent variable in our analyses (see section 3). Faces with greater key press scores are those with greater motivational salience (Aharon et al., 2001).

\subsection{Hormonal Assays}

Saliva samples were frozen immediately and stored at $-32^{\circ} \mathrm{C}$ until being shipped, on dry ice, to the Salimetrics Lab (Suffolk, UK) for analysis. Participants were instructed to avoid consuming alcohol and coffee in the 12 hours prior to participation and avoid eating, drinking, chewing gum or brushing their teeth in the 60 minutes prior to participation. Samples were assayed by Salimetrics using the Salivary $17 \beta$-Estradiol Enzyme Immunoassay Kit 1-3702 (M=4.27 pg/mL, SD=1.07 pg/mL), Salivary Progesterone Enzyme Immunoassay Kit 1-1502 (M=148.82 pg/mL, SD=65.63 $\mathrm{pg} / \mathrm{mL}$ ), and Salivary Testosterone Enzyme Immunoassay Kit 1-2402 $(\mathrm{M}=82.99 \mathrm{pg} / \mathrm{mL}, \mathrm{SD}=21.25 \mathrm{pg} / \mathrm{mL})$. All assays passed Salimetrics' quality 
control. Estradiol, progesterone, and testosterone were assayed because changes in these hormones have been implicated in studies of within-woman changes in perceptual judgments of faces (reviewed in Roney et al., 2011). We also calculated estradiol-to-progesterone ratio $(M=0.03, S D=0.02)$ from women's estradiol and progesterone data because estradiol-to-progesterone ratio is correlated with fertility (Landgren et al., 1980; Baird et al., 1991) and some researchers have suggested that women's responses to facial cues may covary with estrogen-to-progesterone ratio (e.g., Frost, 1994).

\subsection{Analyses}

We used multilevel analyses with cross-classified structures to test for withinsubject effects of hormone levels on the motivational salience of faces. Separate analyses were carried out for attractiveness and sexual dimorphism. All continuous predictors were centered on their grand means. Key-press scores served as our dependent variable in both analyses and both analyses were conducted using R (R Core Team, 2013), Ime4 (Bates et al., 2014), and ImerTest (Kuznetsova et al., 2013). In both sets of analyses, random effects of session nested within participant, face, and the interaction between participant and face were included. The equations and full results for each model are given in our Supplemental Materials.

\section{Results}

\subsection{Attractiveness}

Testosterone, estradiol, progesterone, and estradiol-to-progesterone ratio were entered for each participant's test session to test for independent within- 
subject effects of the different hormone measures on key-press scores. Facial attractiveness and sex of face $(0=$ female, $1=$ male $)$ were entered for each face (see the section 2.2 for details of these ratings). Interactions between facial attractiveness and each of the hormone measures, between sex of face and each of the hormone measures, between facial attractiveness and sex of face, and among facial attractiveness, sex of face and each of the hormone measures were also included in our initial model.

This initial analysis revealed no three-way interactions among facial attractiveness, sex of face and any of the hormone measures (all $|\mathrm{t}|<1.30$, all $p>$.19), except for estradiol-to-progesterone ratio $(t=-2.22, p=.027)$. There were no significant two-way interactions between sex of face and any of the hormone measures or facial attractiveness (all $|t|<1.57$, all $p>.11$ ). The effect of facial attractiveness interacted with testosterone $(t=5.71, p<.001)$ and estradiol-to-progesterone ratio $(\mathrm{t}=2.43, \mathrm{p}=.015)$, but not estradiol or progesterone (both $|t|<0.82$, both $p>.41$ ).

To interpret these results, all non-significant interactions were removed from the model. The three-way interaction among facial attractiveness, sex of face, and estradiol-to-progesterone ratio was significant in this reduced model $(\mathrm{t}=$ 2.75, $p=.006)$. For female faces, there was a significant positive effect of facial attractiveness $(\mathrm{t}=9.43, \mathrm{p}<.001)$, confirming that more attractive female faces generally had greater motivational salience, and no effect of estradiolto-progesterone ratio $(\mathrm{t}=-0.14, \mathrm{p}=.89)$. However, the effect of female facial attractiveness on key-press scores was greater in test sessions with higher 
estradiol-to-progesterone ratio $(t=3.31, p<.001)$. For male faces, there was also a significant positive effect of facial attractiveness $(t=10.72, p<.001)$, and the effect of estradiol-to-progesterone ratio was not significant $(t=0.52, p$ $=.60)$. By contrast with our results for female faces, the effect of male facial attractiveness on key-press scores did not vary as a function of estradiol-toprogesterone ratio $(\mathrm{t}=-0.70, \mathrm{p}=.48)$.

There was also a significant positive effect of testosterone $(t=2.39, p=.018)$, indicating that key-press scores were generally greater in test sessions with higher testosterone levels. These effects were qualified by the significant interaction between facial attractiveness and testosterone $(\mathrm{t}=6.66, \mathrm{p}<.001)$, indicating that the positive effect of facial attractiveness on key-press scores was more pronounced in test sessions with higher testosterone levels. Note that our initial model showed no significant three-way interaction among sex of face, facial attractiveness, and testosterone.

\subsection{Sexual dimorphism}

Our initial model was identical to the initial model in the previous set of analyses, except that sexual dimorphism ratings (see section 2.2 for details of these ratings) were used in place of attractiveness ratings. This initial analysis revealed no three-way interactions among sexual dimorphism, sex of face and any of the hormone measures (all $|t|<1.56$, all $p>.11$ ), except for testosterone $(\mathrm{t}=-4.67, \mathrm{p}<.001)$. There were significant two-way interactions between sexual dimorphism and estradiol-to-progesterone ratio $(\mathrm{t}=2.66, \mathrm{p}=$ $.008)$, sexual dimorphism and testosterone $(t=5.44, p<.001)$, sexual 
dimorphism and sex of face $(\mathrm{t}=-3.31, \mathrm{p}=.001)$, and sex of face and testosterone $(\mathrm{t}=-2.71, \mathrm{p}=.007)$. No other interactions were significant (all $|\mathrm{t}|$ $<0.95$, all $p>$.34).

All non-significant interactions were then removed from the model. In this reduced model, there was a significant interaction between sexual dimorphism and estradiol-to-progesterone ratio $(\mathrm{t}=3.39, \mathrm{p}<.001)$, indicating that sexual dimorphism had a greater effect on key-press scores in test sessions with higher estradiol-to-progesterone ratios. Note that our initial model showed no significant three-way interaction among sex of face, sexual dimorphism, and estradiol-to-progesterone ratio.

The three-way interaction among sexual dimorphism, sex of face, and testosterone that was significant in the full model was also significant in the reduced model $(t=-4.68, p<.001)$. For female faces, there was a significant positive effect of sexual dimorphism $(t=5.09, p<.001)$, showing that more feminine female faces generally had greater motivational salience. There was also a significant positive effect of testosterone $(t=3.38, p<.001)$, indicating that the motivational salience of female faces in general was greater in test sessions with higher testosterone. These effects interacted $(t=5.42, p<$ .001), indicating that the effect of female sexual dimorphism on key-press scores was greater in test sessions with higher testosterone. For male faces, the effect of sexual dimorphism was not significant $(t=0.51, p=.61)$. There was a significant positive effect of testosterone for male faces $(t=2.12, p=$ .035), indicating that the reward value of male faces in general was greater in 
test sessions with higher testosterone. Unlike for female faces, these effects did not interact $(\mathrm{t}=-1.12, \mathrm{p}=.26)$.

Including participant age or session $\operatorname{order}(1,2,3,4,5)$ in our attractiveness and sexual dimorphism models did not alter the pattern of results.

\section{Discussion}

Consistent with the results of previous behavioral (e.g., Hahn et al., 2013) and neuroimaging (see Bzdok et al., 2011 and Mende-Siedlecki et al., 2013 for meta-analytic reviews) studies, physically attractive male and female faces generally had greater motivational salience than relatively unattractive faces. Consistent with previous research suggesting that sexual dimorphism and attractiveness are more strongly and reliably correlated in female faces than male faces (see Rhodes, 2006 for a meta-analytic review), we also found that female, but not male, faces with higher sexual dimorphism generally had greater motivational salience. Our analyses also suggested that the motivational salience of facial attractiveness and sexual dimorphism was modulated by changes in women's hormone levels, however.

The effect of physical attractiveness on the motivational salience of faces interacted with the effect of women's salivary testosterone level. Furthermore, this interaction was not qualified by a higher-order interaction involving sex of face, suggesting that testosterone has similar effects on the motivational salience of attractiveness for male and female faces. Attractiveness had greater positive effects on the motivational salience of faces in test sessions 
where women had higher salivary testosterone levels. This pattern of results was also observed for sexual dimorphism in female faces, but was not observed for sexual dimorphism in male faces. Our results then suggest that women's testosterone levels modulate the motivational salience of facial attractiveness, consistent with the results of studies in which administering testosterone to women increased responses to financial incentives in brain regions implicated in motivation and reward processing (Hermans et al., 2010). Consequently, our data present new, converging evidence that testosterone plays a potentially important role in reward sensitivity (McCall \& Singer, 2012). Some prior work suggests that viewing faces in general is rewarding (e.g., Kawabata \& Zeki, 2008). This being the case, that we found the motivational salience of faces in general to be greater when testosterone levels were high also supports McCall and Singer's (2012) proposal.

The effect of sexual dimorphism on the motivational salience of faces interacted with the effect of women's estradiol-to-progesterone ratio. Moreover, this interaction was not qualified by a higher-order interaction involving sex of face, suggesting that estradiol-to-progesterone ratio has similar effects on the motivational salience of sexual dimorphism for male and female faces. Faces with high sexual dimorphism had greater motivational salience when estradiol-to-progesterone ratio was high. A similar pattern of results was also evident for female, but not male, facial attractiveness. Given strong associations between estradiol-to-progesterone ratio and conception risk (Landgren et al., 1980; Baird et al., 1991), these results are consistent with other research suggesting that women's attraction to masculine men is 
greater when conception risk is high (see Gildersleeve et al., 2014 for discussion). Our results also suggest that women may be more sensitive to female attractiveness at this time. That attractive, feminine female faces have greater motivational salience to women when their estradiol-to-progesterone ratio is high is, perhaps, surprising, given that some previous research has suggested that women derogate the attractiveness of other women when conception risk is high (Fisher, 2004). That attractive, feminine female faces have greater motivational salience to women when their estradiol-toprogesterone ratio is high suggests that women do not necessarily increase avoidance of attractive competitors for mates when conception risk is high. We speculate here that greater motivational salience for attractive, feminine female faces when estradiol-to-progesterone ratio is high may function to facilitate enhanced monitoring of attractive competitors and/or modeling of those competitors' behaviors at points in the menstrual cycle when women are thought to be more likely to compete for high-quality mates (Fisher, 2004). Estradiol-to-progesterone ratio and testosterone may have different effects on responses to male faces because, while estradiol-to-progesterone ratio is a very good predictor of conception risk across the menstrual cycle (Landgren et al., 1980; Baird et al., 1991), testosterone may be more sensitive to situational factors related to competition for resources and mating (van Anders et al., 2011).

In conclusion, our analyses of salivary hormone levels suggest that the motivational salience of facial attractiveness is modulated by within-woman changes in testosterone levels and, to a lesser extent, estradiol-to- 
progesterone ratios. Previous studies have demonstrated that the motivational salience of attractive faces is variable by showing that other types of facial cue (e.g., emotional expression or gaze direction) can modulate responses to physically attractive versus physically unattractive faces in brain regions involved in motivation and reward processing (Kampe et al., 2001; O'Doherty et al., 2003). Here we present new evidence that the motivational salience of physically attractive faces is variable, finding that within-woman changes in hormone levels also modulate the motivational salience of physically attractive faces. Moreover, these changes in the reward value of facial attractiveness may contribute to changes in women's actual behavior towards physically attractive and unattractive individuals during the menstrual cycle (e.g., Senior et al., 2007; Lucas \& Koff, 2013).

\section{Acknowledgments}

This research was funded by Economic and Social Research Council Grant ES/I031022/1 and European Research Council Grant 282655.

\section{References}

Aharon, I., Etcoff, N., Ariely, D., Chabris, C.F., O'Connor, E., Breiter, H.C., 2001. Beautiful faces have variable reward value: $f M R I$ and behavioral evidence. Neuron 32, 537-551.

Baird, D.D., Weinberg, C.R., Wilcox, A.J., McConnaughey, D.R., Musey, P.I., 1991. Using the ratio of urinary oestrogen and progesterone metabolites to estimate day of ovulation. Stat. Med. 10, 255-266. 
Bao, A.-M., Liu, R.-Y., Van Someren, E., Hofman, M.A., Cao, Y.-X., Zhou, J.N., 2003. Diurnal rhythm of free estradiol during the menstrual cycle. Eur. J. Endocrinol. 148, 227-232.

Bates, D., Maechler, M., Bolker, B., Walker, S., 2014. Ime4: Linear mixedeffects models using Eigen and S4. R package version 1.0-6. http://CRAN.R-project.org/package=Ime4

Bobst, C., Sauter, S., Foppa, A., Lobmaier, J.S., 2014. Early follicular testosterone level predicts preference for masculinity in male faces-But not for women taking hormonal contraception.

Psychoneuroendocrinology, 41, 142-150.

Bzdok, D., Langner, R., Caspers, S., Kurth, F., Habel, U., Zilles, K., Laird, A., Eickhoff, S.B., 2011. ALE meta-analysis on facial judgments of trustworthiness and attractiveness. Brain Struct. Funct. 215, 209-223.

Dreher, J.-C., Schmidt, P.J., Kohn, P., Furman, D., Rubinow, D., Berman, K.F., 2007. Menstrual cycle phase modulates reward-related neural function in women. Proc. Natl. Acad. Sci. 104, 2465-2470.

Fisher, M., 2004. Female intrasexual competition decreases female facial attractiveness. Proc. R. Soc. Lond. B. 271, S283-S285.

Frost, P., 1994. Preference for darker faces in photographs at different phases of the menstrual cycle: preliminary assessment of evidence for a hormonal relationship. Percept. Motor Skill. 79, 507-514.

Gildersleeve, K., Haselton, M.G., Fales, M.R., 2014. Do women's mate preferences change across the ovulatory cycle? A meta-analytic review. Psychol. Bull. in press. doi: 10.1037/a0035438 
Hahn, A.C., Xiao, D., Sprengelmeyer, R., Perrett, D.I., 2013. Gender differences in the incentive salience of adult and infant faces. Q. J. Exp. Psychol. 66, 200-208.

Hermans, E.J., Bos, P.A., Ossewaarde, L., Ramsey, N.F., Fernández, G., Van Honk, J., 2010. Effects of exogenous testosterone on the ventral striatal BOLD response during reward anticipation in healthy women. Neuroimage 52, 277-283.

Kampe, K.K., Frith, C.D., Dolan, R.J., Frith, U., 2001. Reward value of attractiveness and gaze. Nat. 413, 589-589.

Kawabata, H., Zeki, S., 2008. The neural correlates of desire. PLoS ONE 3, e3027.

Kuznetsova, A., Bruun Brockhoff, P.,Haubo Bojesen Christensen, R., 2013. ImerTest: Tests for random and fixed effects for linear mixed effect models (Imer objects of Ime4 package). R package version 2.0-3. http://CRAN.R-project.org/package=ImerTest

Landgren, B.-M., Unden, A.-L., Diczfalusy, E., 1980. Hormonal profile of the cycle in 68 normally menstruating women. Acta Endocrinol. 94, 89-98.

Langlois, J.H., Kalakanis, L., Rubenstein, A.J., Larson, A., Hallam, M., Smoot, M., 2000. Maxims or myths of beauty? A meta-analytic and theoretical review. Psychol. Bull. 126, 390-423.

Levy, B., Ariely, D., Mazar, N., Chi, W., Lukas, S., Elman, I., 2008. Gender differences in the motivational processing of facial beauty. Learn. Motiv. 39, 136-145. 
Lucas, M., Koff, E., 2013. How conception risk affects competition and cooperation with attractive women and men. Evol. Hum. Behav. 34, 1622.

Macrae, C.N., Alnwick, K.A., Milne, A.B., Schloerscheidt, A.M., 2002. Person perception across the menstrual cycle: Hormonal influences on socialcognitive functioning. Psychol. Sci. 13, 532-536.

McCall, C., Singer, T., 2012. The animal and human neuroendocrinology of social cognition, motivation and behavior. Nat. Neurosci. 15, 681-688.

Mende-Siedlecki, P., Said, C.P., Todorov, A., 2013. The social evaluation of faces: a meta-analysis of functional neuroimaging studies. Soc. Cogn. Affect. Neurosci. 8, 285-299.

O’Doherty, J., Winston, J., Critchley, H., Perrett, D., Burt, D.M., Dolan, R.J., 2003. Beauty in a smile: the role of medial orbitofrontal cortex in facial attractiveness. Neuropsychologia 41, 147-155.

Papacosta, E., Nassis, G.P., 2011. Saliva as a tool for monitoring steroid, peptide and immune markers in sport and exercise science. J. Sci. Med. Sport 14, 424-434.

R Core Team, 2013. R: A language and environment for statistical computing. R Foundation for Statistical Computing, Vienna, Austria. URL http://www.R-project.org/.

Rhodes, G., 2006. The evolutionary psychology of facial beauty. Annu. Rev. Psychol. 57, $199-226$

Roney, J.R., Simmons, Z.L., 2008. Women's estradiol predicts preference for facial cues of men's testosterone. Horm. Behav. 53, 14-19. 
Roney, J.R., Simmons, Z.L., Gray, P.B., 2011. Changes in estradiol predict within-women shifts in attraction to facial cues of men's testosterone. Psychoneuroendocrinology 36, 742-749.

Senior, C., Lau, A., Butler, M.J., 2007. The effects of the menstrual cycle on social decision making. Int. J. Psychophysiol. 63, 186-191.

van Anders, S.M., Goldey, K.L., Kuo, P.X., 2011. The steroid/peptide theory of social bonds: integrating testosterone and peptide responses for classifying social behavioral contexts. Psychoneuroendocrinology 36, $1265-1275$.

Veldhuis, J.D., Christiansen, E., Evans, W.S., Kolp, L.A., Rogol, A.D., Johnson, M.L., 1988. Physiological profiles of episodic progesterone release during the midluteal phase of the human menstrual cycle: Analysis of circadian and ultradian rhythms, discrete pulse properties, and correlations with simultaneous luteinizing hormone release. J. Clin. Endocrinol. Metab. 66, 414-421.

Welling, L.L., Jones, B.C., DeBruine, L.M., Conway, C.A., Law Smith, M., Little, A., Feinberg, D.R., Sharp, M.A., Al-Dujaili, E.A., 2007. Raised salivary testosterone in women is associated with increased attraction to masculine faces. Horm. Behav. 52, 156-161. 